\title{
The Incidence and Risk Factors of Nosocomial Infections in ICU
}

\author{
Veres Mihaly, Benedek Orsolya*, Orlandea Monica, Perenyi Anett Anna, Katona Hajna, Copotoiu Sanda \\ Maria, Kovacs Judit
}

University of Medicine and Pharmacy Tirgu Mures, România

\begin{abstract}
Introduction: The increased incidence of nosocomial infections in intensive care units, with frequent occurrence of multiresistant pathogens increase mortality and often raises therapeutic problems. Objectives: to assess the incidence of nosocomial infections, and risk factors. Methods: The study includes 125 patients hospitalized in the Clinic of Anesthesiology and Intesive Care in the Emergency County Hospital and Cardiovascular Surgery Targu Mures. The patients were divided into two groups: the control group ( $n=99)$, patients who did not develop infections during hospitalization in the ICU and the group with infection $(n=26)$. Results: The incidence of nosocomial infections in our intensive care unit was $19.1 \%$, the most common pathogen being Acinetobacter baumanii. There were no significant differences between the two groups regarding demographic data, the most important risk factor was chronic alcohol consumption. SAPS II. and SOFA scores showed higher values in the group with infection on the day of admission. This group showed lower levels of arterial blood oxygen (Horowitz index), lower sodium level, and higher number of platelets compared to the control group. The mortality in the group with infection was $47.65 \%$ compared to the control. Conclusions: Nosocomial infections in critically ill patients are associated with hypoxemia, thrombocytopenia, hyponatremia and a bad outcome.
\end{abstract}

Keywords: hypoxemia, sodium level, platelets

Received: 08 September 2015 / Accepted: 05 March 2016

\section{Introduction}

Nosocomial infections are infections acquired in hospitals or other healthcare facilities. The patients must have no signs of infection at the admission. In the intensive care unit patients are frequently exposed to infection, many of them attributed to antimicrobial-resistant pathogens. The incidence of these infections varies between $2.3-49.2 \%$ on intensive care. According to the definition, nosocomial infections develop after 48 hours from admission in hospital, the incidence being variable depending on the country, hospital and medical unit [1,2].

Nosocomial infections significantly prolong the length of stay in intensive care and increase morbidity, mortality (up to $50 \%$ ). The main therapeutic points of the nosocomial infections are rapid detection, effective treatment and appropriate prevention. There is a protocol regarding the diagnosis of nosocomial infections named HAIICU (Hospitals in Europe Link for Infection Control through Surveillance), described by the European Center for Disease Prevention and Control [2].

The most common manifestations of nosocomial infections are: pneumonia, urinary tract infections, followed by systemic infections [3]. The highly incriminated pathogens are: Staphylococcus aureus, Pseudomonas species, Enterobacteriaceae and fungi (Candida species) [4]. Nosocomial infections in intensive care are mainly related to central catheter, urinary tract infections and ventilator-associated pneumonia [2].

* Correspondence to: Orsolya Benedek

E-mail: benedek.orsolya@umftgm.ro
Hospital-Acquired Pneumonia (HAP) occur within 48 hours from hospital admission [5]. Some of these are associated to mechanical ventilation (ventilation-associated pneumonia-VAP). The ventilator associated pneumonia mortality is up to $50 \%$ [6]. The risk factors of VAP are: age (older than 70 years), chronic lung disease, reduced consciousness and aspiration [7]. The detection of pathogens is obtained from the trachea suctioning and bronchoalveolar lavage (BAL), done preferably before starting antibiotic therapy. The efficacy of the treatment depends on early initiation of therapy with large spectrum antibiotics followed by targeted therapy, according to the bacteriological results [8].

Risk factors for central line-associated blood stream infection (CLABSI) are old age, immunosuppression, malnutrition, parenteral nutrition, burns. The femoral catheter (followed by internal jugular, subclavian vein catheters placed) predispose for infections [2]. Insertion site of central venous catheter-related infections can be classified in three categories, local, systemic infections and sepsis caused by central venous catheter (CVC) which can occur before or after 48 hours of catheter removal. The cultures from CVC will be positive two hours faster than the peripheral. Risk factors include old age, immunosuppression, malnutrition, parenteral nutrition, burns. The pathogen most commonly met is Staphylococcus Aureus [9].

Catheter associated urinary tract infections (CA-UTI) occur after more than 48 hours of urethral catheterization. A distinction should be made between catheter associated asymptomatic bacteriuria and symptomatic forms. The predisposing factors for infections are prolonged use of 
urinary catheters. The targeted therapy must be initiated after positive urine culture [10].

Wound infections appear after surgical intervention within 30 days. The most common pathogens causing these infections are Enterococci, Staphylococci and coagulase-negative species of Enterobacter [11]. The incidence can reach $38 \%$. The most important predisposing factors are diabetes and malnutrition [12]. Enterocolitis caused by Clostridium difficile is spread by direct or indirect contact, and has an increasing trend in the recent years, therefore require special attention to prevent its spread. Infections caused by Clostridium difficile are more common in older people with multiple antimicrobial therapy, in patients receiving chemotherapy and proton pump inhibitors, in kidney failure and gastrointestinal surgery $[13,14]$.

Our objectives were to assess the incidence of nosocomial infections and to identify the risk factors.

\section{Methods}

We performed a prospective, clinical observational study. We enrolled 125 consecutive patients hospitalized between $1^{\text {st }}$ October $2014-30^{\text {th }}$ of April 2015 at Anesthesia and Intensive Care Units at Emergency County Hospital and in the Cardiovascular Surgery Targu Mures.

We included all patients who did not show any laboratory signs of infection, as evidenced by negative cultures from blood, urine and tracheal secretions. Patients were divided into two groups: the control group $(\mathrm{n}=99)$ including patients who did not develop infections during hospitalization in intensive care and the group with infection comprising patients with different kind of hospital acquired infections ( $\mathrm{n}=26)$.

The patients were grouped taking in to consideration the type of their basic illness, forming the groups of medi$\mathrm{cal}$ and surgical patients. We analyzed the patients' demographic data, associated diseases, alcohol or tobacco use and body temperature. Cardiovascular status assessed by invasive monitoring of systolic, diastolic and mean blood pressure values, pulse and standard ECG. Respiratory and ventilation parameters were registered in spontaneous breathing when an oxygen face mask was needed and in mechanical ventilation the ventilator parameters as $\mathrm{FiO} 2$, assisted or controlled ventilation modes were also noted. The Horowitz coefficient was calculated for each patient. Blood gas analysis was performed for each patient, recording $\mathrm{pH}, \mathrm{pO}_{2}, \mathrm{pCO}_{2}$, lactate, sodium bicarbonate. Hemoglobine, hematocrit, leucocyte count, thrombocyte count was evidenced by a full blood count. The possibility of onset of an organ dysfunction was monitored using urea and creatinine for the kidney and aspartate aminotransferase, alanine aminotransferase, bilirubin for the liver. Neurological status was assessed daily by the use of the Glasgow Coma Score. The data was collected from all patients on admission and in patients with evidence of infection on the day of positive bacteriological culture. At admission SAPS II. (Simplified Acute Physiology Score) and SOFA
(Sequential Organ Failure Assessment) were calculated. We followed the bacteriological data from tracheal tubes (broncho-alveolar lavage), arterial and venous (central and peripheral) catheters, their length of use. In infected patients pathogenic bacteria, antibiotic sensitivity, and localization of the infection were recorded. Distinction between colonization and infection was set by the HAIICU (Hospitals in Europe Link for Infection Control through Surveillance), described by the European Center for Disease Prevention and Control. definitions.

Our results were introduced in Microsoft Office Excel 2007 and the statistical analysis was realized with the GraphPad Prism 5.0 software. We compared in this two groups the averages of parameters using t-test and the analysis of contingency tables was made with Fisher's exact test. For both tests we determined a $\mathrm{p}$ value, and we stated, if $\mathrm{p}$ $<0.05$, the differences are statistically significant.

\section{Results}

The incidence of nosocomial infection in our study was 19.1\%. The multiresistant Acinetobacter baumanii was the most common bacteria contracted by 10 patients. Out of this group in 11 cases it was isolated from trachea-bronchial secretions, 1 cases of central venous line infection. The second most common germ was Clostridium difficile, in 8 patients, all isolated from fecal culture. ESBL (Extended Spectrum Beta -Lactamase)-producing Klebsiella pneumoniae was present in 5 patients, isolated from tracheabronchial secretions and in 1 case from surgical wound . Further bacteria were Serratia marcescens ( 2 cases, isolated from trachea-bronchial secretions), Methicillin-Resistant Staphylococcus aureus ( 2 cases of central venous line infection), Escherichia coli (1 case, isolated from trachea-bronchial secretions), Candida albicans (1 case, isolated from trachea-bronchial secretions), Candida non-albicans (1 case, isolated from trachea-bronchial secretions), Methicillin-susceptible Staphylococcus aureus (1 case, isolated from trachea-bronchial secretions), Enterococcus faecalis (1 case, isolated from trachea-bronchial secretions), Enterobacter asburiae (1 case, isolated from trachea-bronchial secretions), Providencia stuartii (1 case, isolated from tracheabronchial secretions). The most common infections caused by these bacteria were the following: pneumonia $80.95 \%$ equivalent of 20 infections and 6 colonisations; enterocolitis infectiosa $28.57 \%$ equivalent of 8 infections; cannula sepsis $14.28 \%$ translating in 3 infections; wound infection $4.76 \%$ representing 1 case. There was no significant difference between the two groups with respect to gender although the man rate for the control group was $42.4 \%$ and for the infected patients was $15.2 \%(\mathrm{p}=0.0844)$, the female rate for the control group was $36,8 \%$ and for the infected patients was $5.6 \%(\mathrm{p}=0.0756)$. In the control group the average age was $61.06 \pm 1.3$ years and $60.14 \pm 3.102$ years in the infected group $(\mathrm{p}=0.7657)$.

The average body-mass index in the control group was $27.92 \pm 0.5949 \mathrm{~kg} / \mathrm{m} 2$ and in the infected group it 
was $30.07 \pm 1.581 \mathrm{~kg} / \mathrm{m} 2(\mathrm{p}=0.1623)$. The most common comorbidity in both groups was diabetes mellitus: $29.21 \%$ in control group, $42.85 \%$ in patients with infections $(\mathrm{p}=0.2976)$. This was followed by chronic obstructive pulmonary disease: control group $15.73 \%$, infected group $19.04 \%$ ( $\mathrm{p}=0.7455)$. There were no significant differences between the two groups with respect to smoking $(\mathrm{p}=0.2470)$, control group $20.22 \%$; infected patients $33.33 \%)$, the incidence of chronic alcohol abuse in the control group was $4.49 \%$ respectively in infected patients $28.57 \%(\mathrm{p}=0.0031)$. (Table I).

The infections were more frequent in patients who were admitted through internal medicine. In the group of patients with infections, $43.01 \%$ came from medical units. Patients without infections were from surgical units, representing $66.35 \%(\mathrm{p}=0.0006)$.

There were no significant differences between the groups regarding the mean heart rate at admission to the intensive care unit (ICU) $(\mathrm{p}=0.8663)$. Analyzing the mean arterial blood pressure, we found that in the control group it was $78.19 \pm 23.083 \mathrm{Hg} \mathrm{mm}$ and in the infected patients $62.04 \pm 22.449 \mathrm{Hg} \mathrm{mm}(\mathrm{p}=0.0092)$. Arrhythmias (atrial-, ventricular flutter/ fibrillation, fascicular block) occurred more frequently in the infected patients: $38.09 \%$ versus $17.97 \%(\mathrm{p}=0.0741)$.

The mean core body temperature measured on the day of admission to ICU in control group was $36.77 \pm 0.0784$ Celsius degrees and in the group with infection it was $37.21 \pm 0.01541$ Celsius degrees. There was no significant difference between the groups for the $\mathrm{pH}$ and lactate levels on admission, however in the infected group on the first day of admission, a significantly lower sodium level $(\mathrm{p}=0.0101)$ was measured (Table II).

For the assessment of appropriate oxygenation we used the Horowitz quotient, which is defined as the ratio of partial pressure of oxygen in arterial blood and the fraction of inspired oxygen. On the day of admission, the mean Horowitz qoutient in the control group was $316.4 \pm 20.35$ while in the infected patients it was significantly lower, $190.6 \pm 26.85(\mathrm{p}=0.0023)$. In the first 24 hours kidney and liver function did not show any pathological changes. The number of white blood cells

Table I. Demographic data, associated diseases

\begin{tabular}{lccc}
\hline & Control group $(\mathrm{n}=99)$ & Infected group $(\mathrm{n}=26)$ & $\mathrm{P}$ value \\
\hline Age & $61.06 \pm 1.3$ & $60.14 \pm 3.102$ & 0.7657 \\
Sex & & & \\
$\quad$ Male & $42.4 \%$ & $15.2 \%$ & 0.0844 \\
$\quad$ Female & $36.8 \%$ & $5.6 \%$ & 0.0756 \\
BMI $(\mathrm{kg} / \mathrm{m} 2)$ & $27.92 \pm 0.5949$ & $30.07 \pm 1.581$ & 0.1623 \\
Alcohol use & $4.49 \%$ & $28.57 \%$ & 0.0031 \\
Tabacco & $20.22 \%$ & $33.33 \%$ & 0.2470 \\
Diabetes & $29.21 \%$ & $42.85 \%$ & 0.297 \\
COPD & $15.73 \%$ & $19.04 \%$ & 0.7455 \\
\hline
\end{tabular}

BMI- Body Mass Index, COPD- Chronic Obstructive Pulmonary Disease on the first day of the ICU was slightly higher in both groups: control group $11.73 \pm 0.68^{*} 10^{3} / \mu$ l versus infected group $12.54 \pm 1.12^{*} 10^{3} / \mu \mathrm{l}(\mathrm{p}=0.645)$, hemoglobine level (control group $10.51 \pm 0.3012 \mathrm{~g} / \mathrm{dl}$ versus infected group $10.99 \pm 0.5661 \mathrm{~g} / \mathrm{dl}, \mathrm{p}=0.4559)$ were slightly decreased. The number of platelets, although in normal range, was higher in the group with infection (Table III).

The mean value Glasgow Coma Score GCS in the control group was $13.55 \pm 1.18$ points in contrast with the infected patients, where it was $7.71 \pm 1.76$ points $(\mathrm{p}<0.0001)$. To measure the severity of organ dysfunctions at admission, we used the SAPS II. score. In the control group the median SAPS II. score was $35.19 \pm 5.789$ points and in the infected group $56.12 \pm 6.874$ points $(\mathrm{p}<0.0001)$, with the resulted predictive mortality in the control group $27.32 \%$, and in the experimental group 65.4\% $(\mathrm{p}<0.0001)$. The median SOFA score in the control group was $5.122 \pm 2.643$ points with a resulted predictive mortality of $14.81 \%$ and in the group with infections $7.42 \pm 2.027$ points, with a resulted predictive mortality of $24.35 \%$ ( $\mathrm{p}=0.0009$; $\mathrm{p}=0.0014$ ). (Figure 1, Figure 2).

The first infections occurred after 7.5 days. Analyzing the length of use of cannulas and catheters during the intensive care treatment, we observed that in the infected group the tracheal tubes were used for a longer period before changing with a mean value of $4.9 \pm 2.53$ days versus $1.8 \pm 0.74$ days in the control group ( $<<0.0001)$. A higher percentage of infected patients had tube replacement $(52.38 \%)$ than non-infected patients $(11.23 \%)(\mathrm{p}=0.0001)$.

The first central venous catheter was changed in 3.4 days in the control group and in 9.3 days in the infected patients $(\mathrm{p}<0.0001)$. There was a significant difference between the two groups regarding the length of use of arterial catheters: in control group $3 \pm 1.602$ days, in the infected group $8.7 \pm 2.368$ days $(\mathrm{p}<0.0001)$.

$66.66 \%$ of the infected patients and $88.76 \%$ of the non-infected patients received Ceftriaxon $(\mathrm{p}=0.0193)$. The length of administration was $3.4 \pm 0.336$ days in the control group, versus $9.4 \pm 1.652$ days in the infected patients $(\mathrm{p}<0.0001)$. Patients without infection received antibiotics with on average 1.7 and the infected group 5 (Ceftriaxon, Ciprofloxacin, Levofloxacin, Meropenem, Polymyxin

Table II. Disturbances of the hydroelectrolytic and acid-base homeostasis

\begin{tabular}{lccc}
\hline & Control group & Infected group & $\mathrm{p}$ \\
\hline $\mathrm{pH}$ & $7,34 \pm 0,01$ & $7,29 \pm 0,02$ & 0,1008 \\
Lactate $(\mathrm{mmol} / \mathrm{l})$ & $5,45 \pm 1,24$ & $9,91 \pm 3,34$ & 0,1296 \\
Sodium Bicarbonate $(\mathrm{mmol} / \mathrm{l})$ & $22,55 \pm 0,77$ & $22,38 \pm 1,15$ & 0,1719 \\
Sodium (mmol/l) & $142,5 \pm 0,53$ & $133,9 \pm 6,04$ & 0,0101 \\
\hline
\end{tabular}

Table III. Blood count

\begin{tabular}{lccc}
\hline & Control group & Infected group & $\mathrm{p}$ \\
\hline Leukocyte $\left({ }^{*} 103 / \mu \mathrm{l}\right)$ & $11,73 \pm 0,68$ & $12,54 \pm 1,12$ & 0,645 \\
Hemoglobin $(\mathrm{g} / \mathrm{dl})$ & $10,51 \pm 0,3012$ & $10,99 \pm 0,5661$ & 0,4559 \\
Platelets $\left({ }^{*} 103 / \mathrm{ll}\right)$ & $167,3 \pm 8,554$ & $208,7 \pm 21,41$ & 0,0333 \\
\hline
\end{tabular}




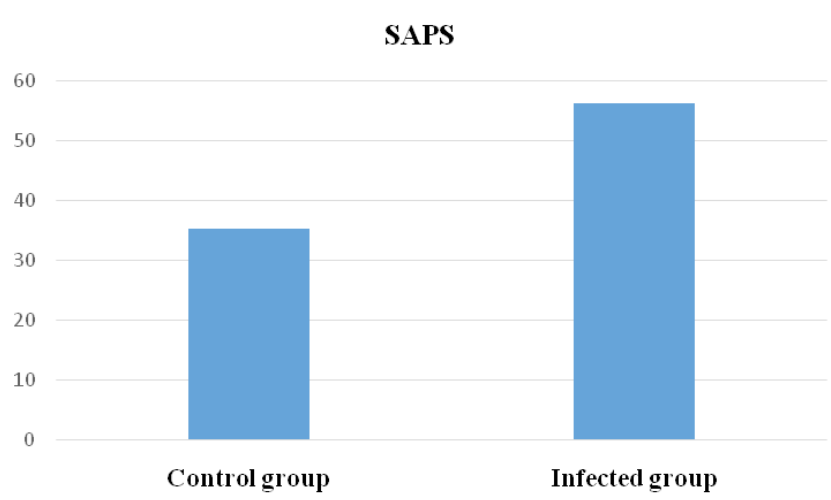

Figure 1. Simplified Acute Physiology Score II- median SAPS II. score in the control group $35.19 \pm 5.789$ points, in the infected group $56.12 \pm 6.874$ points $(p<0.0001)$

E, Imipenem/Cilastatin, Vancomycin, Metronidazol, Amikacin, Tygecyclin, Teicoplanin) $(\mathrm{p}<0.0001)$.

The group with infection had higher mortality $(47.65 \%)$ in contrast with the control group (11.23\%) $(\mathrm{p}=0.0005)$.

\section{Discussion}

The incidence of nosocomial infection in our study $(19.09 \%)$ is the same as in the literature $(2.3-49.2 \%$; $9-37 \%)[1,2]$. Furthermore, the site of infection is the same as in large international studies, the most common is pneumonia followed by urinary infections, cannula sepsis and wound infection. Comparing our results with former published data we observed that there were no significant differences between the infected and non-infected patients regarding demographic data (gender, age and body-mass index). In our patients chronic obstructive pulmonary disease, diabetes and malignant tumors occurred with almost the same incidence, meanwhile other studies show significantly higher rate of infection in the presence of these comorbidities. In our study the incidence of infection was significantly higher in patients with chronic alcohol consumption [3]. Analyzing the cause of hospital admission, we observed that most of the infected patients were admitted to internal medicine and less for elective surgery [3]. A decreased level of sodium was found in the infected patients. Recent studies showed a correlation between hyponatremia (hospital-acquired hyponatremia) and infections (especially with Staphylococcus aureus) [15]. It was also recently proved that a higher level of sodium helped the activation of the immune cells (macrophages), thereby an increased salt consumption may decrease the incidence of infection [16].

In the infected patients, tissue oxygenation was not appropriate at admission, probably the hypoxemia induced metabolic acidosis facilitated the bacterial reproduction by inhibiting the chemotactic movements and the bactericidal capacity of the leukocytes [17]. In addition, these patients often required mechanical ventilation, so the chance for VAP (ventilator associated pneumonia) was higher. At admission in the intensive care unit, all patients received anti-

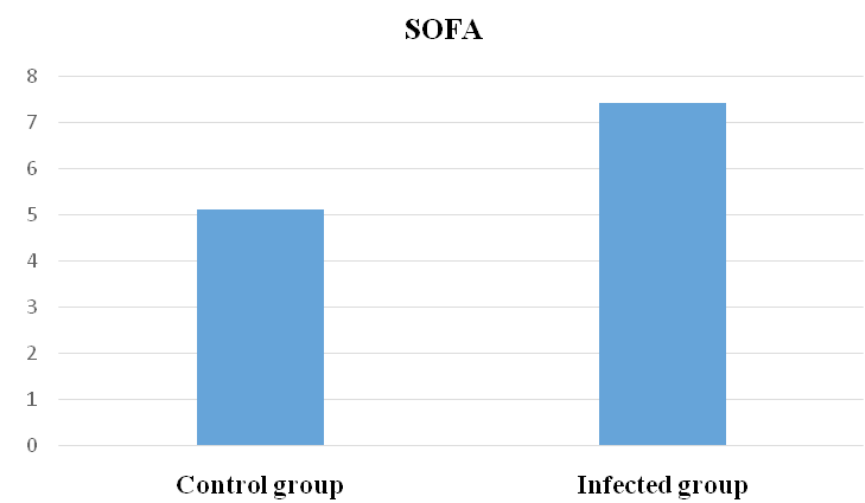

Figure 2. Sequential Organ Failure Assessment Score- median SOFA score in the control group $5.122 \pm 2.643$ points, in the infected group $7.42 \pm 2.027$ points $(0.0009)$

bioprofilaxy (Ceftriaxon). Some studies proved that certain antibiotics, such as Ceftriaxon, Cefotaxim and Meropenem can cause thrombocytosis $[18,19]$. In infections, especially in sepsis, thrombocytopenia occurs because of bone marrow depression caused by inflammatory mediators [20].

Comparing the length of use of orotracheal tubes, it is unequivocal that in the infected patients the orotracheal tube was used for more days. There is a correlation between the long-term intubation, mechanical ventilation and nosocomial pneumonia, especially ventilator-associated pneumonia [21]. There is a similar correlation between the long-term use of central venous cannula and arterial catheter that prolonged time, rare change increases significantly the incidence of nosocomial infection [22]. The studies reveals that more patients in ward may increase the incidence of nosocomial infections (especially if there is infected patient), spread by air and contact which is significant, and also the nurse and medical care may facilitate the transmission of the infections [23].

The reliability and efficiency of predictive scoring systems used were analyzed before. Our results show a significant difference between the two groups regarding the SAPS II. and SOFA scores, similar to other studies [3].

\section{Conclusion}

The incidence of nosocomial infection in the intensive care unit is high, is more frequent if prior chronic alcohol abuse is present. The development of these infections favors patients admitted for medical diseases rather than surgical ones. Lower mean arterial pressure, high body temperature and decreased sodium levels on admission correlate with the presence of a nosocomial infection.

The presence of inappropriate oxygenation and the increased platelet count should raise an alarm. A longer time of use of tracheal tubes, catheters may increase significantly the incidence of nosocomial infections.

\section{Conflicts of interest}

The authors report no conflicts of interest. 


\section{References}

1. Dasgupta S, Das S, Chawan NS, Hazra A - Nosocomial infections in the intensive care unit: Incidence, risk factors, outcome and associated pathogens in a public tertiary teaching hospital of Eastern India. Indian Journal of Critical Care Medicine. 2015;19(1):14-20.

2. Majumdar SS, Padiglione AA - Nosocomial infections in the intensive care unit. Anaesthasia and Intensive Care Medicine. 2012;13(5): 204208

3. Vincent JL, Bihari DJ, Suter PM, Bruining HA, White J, Nicolas-Chanoin $\mathrm{MH}$, Wolff M, Spencer RC, Hemmer M - The prevalence of nosocomial infection in intensive care units in Europe. Results of the European Prevalence of Infection in Intensive Care (EPIC) Study. EPIC International Advisory Committee. The Journal of the American Medical Association. 1995;274(8):639-644.

4. Vincent JL, Rello J, Marshall J, Silva E, Anzueto A, Martin CD, Moreno R, Lipman J, Gomersall C, Sakr Y, Reinhart K - International Study of the Prevalence and Outcomes of Infection in Intensive Care Units. The Journal of the American Medical Association. 2009;302(21):2323-2329.

5. American Thoracic Society, Infectious Diseases Society of America. Guidelines for the management of adults with hospital-acquired, ventilator-associated, and healthcare-associated pneumonia, American Journal of Respiratory and Critical Care Medicine. 2005;171:388-416.

6. Craven DE, Hjalmarson $\mathrm{KI}$ - Ventilator-associated tracheobronchitis and pneumonia: thinking outside the box. Clinical Infectious Diseases. 2010;51(1):59-66.

7. Kollef $\mathrm{MH}$ - Clinical characteristics and treatment patterns among patients with ventilator-associated pneumonia. Chest. 2006;129:12101218

8. Rodriguez JL, Gibbons KJ, Bitzer LG - Pneumonia: incidence, risk factors and outcome in injuried patients. Journal of Trauma-Injury Infections and Critical Care. 1991;31(7):907-914.

9. Mermel LA, Farr BM, Sherertz RJ, Raad II, O'Grady N, Harris JS, Craven DE - Guidelines for the management of intravascular catheter-related infections. Clinical Infectious Diseases. 2001;32:1249-1272.

10. Hooton TM, Bradley SF, Cardenas DD, Colgan R, Geerlings SE, Rice JC, Saint S, Schaeffer Al, Tambayh PA, Tenke P, Nicolle LE - Diagnosis, prevention, and treatment of catheter-associated urinary tract infection in adults: 2009 International Clinical Practice Guidelines from the Infectious Diseases Society of America. Clinical Infectious Diseases. 2010;50:625-663.

11. Jarvis WR, Martone WJ - Predominant pathogens in hospital infections. Journal of Antimicrobial Chemotherapy. 1992;29:19-24.
12. Malone DL, Geunti T, Tracy JK, Gannon C, Napolitano LM - Surgical site infections: reanalysis of risk factors. Journal of Surgical Research. 2002;103(1):89-95.

13. Samore $\mathrm{MH}$, Venkataraman L, DeGirolami PC, Arbeit RD, Karchmer AW - Clinical and molecular epidemiology of sporadric and clustered cases of nosocomial Clostridium difficile diarrhea. The American Journal of Medicine. 1996;100(1): 32-40.

14. Marra AR, Edmond MB, Wenzel RP, Bearman ML - Hospital-aquired Clostridium difficileassociated disease in the intensive care unit setting: epidemiology, clinical course and outcome. BMC Infectious Diseases. 2007;7:42-48

15. Jensen AG, Wachmann CH, Poulsen KB, Espersen F, Scheibel J, Skinhoj $\mathrm{P}$, Frimodt-Moller N -Risk factors for hospital-hquired Staphylococcus aureus bacteremia. Archives of Internal Medicine. 1999; 159(13):14371444

16. Jantsch J, Schatz V, Friedrich D, Schröder A, Kopp C, Siegert I, Maronna A, Wendelborn D, Linz P, Binger KJ, Gebhardt M, Heinig M, Neubert P, Fischer F, Teufel S, David JP, Neufert C, Cavallaro A, Rakova N, Küper C, Beck FX, Neuhofer W, Muller DN, Schuler G, Uder M, Bogdan C, Luft FC, Titze J - Cutaneous $\mathrm{Na}$ + storage strenghtens the antimicrobial barrier function of the skin and boosts macrophage-driven host defense. Cell metabolism. 2015;21(3):493-501.

17. Lardner $\mathrm{A}$ - The effect of extracellular $\mathrm{pH}$ on immune function. Journal of Leukocyte Biology. 2001; 69:522-530.

18. Álvarez-Lerma F, Alvarez B, Luque P, Ruiz F, Dominguez JM, Quintana E, Sanz-Rodriguez C - Empiric broad-spectrum antibiotic therapy of nosocomial pneumonia in the intensive care unit: a prospective observational study. Critical Care. 2006;10(3):78.

19. Mohr FJ - Update on efficacy and tolerability of Meropenem in the treatment of serious bacterial infections. Clinical Infectious Diseases. 2008;47(1): 41-51.

20. Sekhon SS, Roy V - Thrombocytopenia in adults: A practica approach to evaluation and management. Southern Medical Journal. 2006;99(5):491-498.

21. Torres A, El-Ebiary M, Rano A - Respiratory infection complications in the intensive care unit. Clinics in Chest Medicine. 1999;20(2):287-301.

22. Esteve F, Pujol M, Limon E - Blodstream infection related to catheter connections: a prospective trial of two connection systems. Journal of Hospital Infection. 2007;67(1):30-34

23. Pittet D - Improving compliance with hand hygiene in hospitals. Infection Control. 2000;20(6):381386. 